\title{
New convergence theorems for maximal monotone operators in Banach spaces
}

\author{
Siwaporn Saewan \\ Department of Mathematics and Statistics \\ Faculty of Science, Thaksin University \\ Phatthalung, Thailand, 93110 \\ Email: si_wa_pon@hotmail.com
}

Received: February 12, 2021. Revised: March 5, 2021. Accepted: March 15, 2021. Published: March 29, 2021.

\begin{abstract}
The purpose of this paper is to introduce a new hybrid iterative scheme for resolvents of maximal monotone operators in Banach spaces by using the notion of generalized $f$ projection. Next, we apply this result to the convex minimization and variational inequality problems in Banach spaces. The results presented in this paper improve and extend important recent results in the literature.
\end{abstract}

\section{INTRODUCTION}

Maximal monotone operators have frequently proven to be a key class of objects in Optimization and Analysis. Constructing iterative algorithms to approximate zeros of maximal monotone operators is a very active topic in applied mathematics. The problem for finding a zero point of a maximal monotone operator is defined as follows : Given a Banach space $E$ and a maximal monotone operator $T$, we consider the problem for finding a point $u \in E$ such that:

$$
0 \in T(u) \text {. }
$$

The set of all points $u \in E$ such that $0 \in T(u)$ denote by $T^{-1} 0$. This problem is very important in optimization theory and related fields. For example, if $F: E \rightarrow(-\infty, \infty]$ is a proper lower semicontinuous convex function. In this case, the equation $0 \in \partial F(u)$ is equivalent to the problem of minimizing $F$ over $E$.

The proximal point algorithm (PPA) is one of the popular methods for solving (1), which was first proposed by Martinet [1] and further developed by Rockafellar [2] in a framework of maximal monotone operators in a Hilbert space. A variety of problems, for exmaple, convex programming and variational inequalities can be formulated as finding a zero point of maximal monotone operators. Many research study and extend the proximal methods for various models (see, for example, [3], [4] and [5]). Both numerical experiments and theoretical analysis have demonstrated that the PPA is robust and has nice convergence properties. The proximal point algorithm (PPA) according to Rockafellar [2] generates a sequence $\left\{x_{n}\right\}$ via the rule

$$
x_{0} \in H, \quad x_{n+1}=J_{r_{n}} x_{n}, \quad n=1,2,3, \ldots,
$$

where $J_{r_{n}}=\left(I+r_{n} T\right)^{-1}$ and $\left\{r_{n}\right\} \subset(0, \infty)$, then the sequence $\left\{x_{n}\right\}$ converges weakly to an element of $T^{-1}(0)$.

If $T=\partial F$ where $F: E \rightarrow(-\infty, \infty]$ is a proper lower semicontinuous convex function, then (2), is reduced to

$x_{n+1}=\operatorname{argmin}_{y \in H}\left\{F(y)+\frac{1}{2 r_{n}}\left\|x_{n}-y\right\|^{2}, \quad n=1,2,3, \ldots\right.$.
To get the results of strong convergence, Solodov and Sviater [4] modified the proximal point algorithm and projection in a Hilbert space. In 2003, Kohsaka and Takahashi [13] obtained a strong convergence theorem for maximal monotone operators in a Banach space, which extended the result of Solodov and Svaiter in a Hilbert space.

On the other hand, Alber [6], [7] introduced and studied the generalized projections $\pi_{C}: E^{*} \rightarrow C$ and $\Pi_{E}: E \rightarrow C$ in uniformly convex and uniformly smooth Banach spaces. In 2005, Li [9] extended the generalized projection operator from uniformly convex and uniformly smooth Banach spaces to reflexive Banach spaces. Later, Wu and Huang [10] introduced a new generalized $f$-projection operator in Banach spaces. They extended the definition of the generalized projection operators introduced by Abler [7] and proved some properties of the generalized $f$-projection operator. In 2009, Fan et al. [11] presented some basic results for the generalized $f$ projection operator, and discussed the existence of solutions and approximation of the solutions for generalized variational inequalities in noncompact subsets of Banach spaces. Recently, Li et al. [12] proved some property of the generalized $f$ projection operator and proved strong convergence theorems for relatively nonexpansive mappings in Banach spaces.

In 2005, Matsushita and Takahashi [14] proposed the following hybrid iteration method (it is also called the CQ method) with generalized projection for relatively nonexpansive mapping in a Banach space $E$. They obtained a strong convergence theorem for relatively nonexpansive mapping in a Banach space. In 2010, Li et al. [12] introduced and proved the strong convergence theorem for approximation of fixed point of relatively nonexpansive mapping using the properties of generalized $f$-projection operator in a uniformly smooth real Banach space which is also uniformly convex. We remark here that the results of $\mathrm{Li}$ et al. [12] extended and improved on the results of Matsushita and Takahashi [14]. Recently, Saewan and Kumam [15] extended the ideal of the generalized $f$ projection operator to hybrid Ishikawa iteration process for finding a common element of the fixed point set for two countable families of weak relatively nonexpansive mappings and the set of solutions of finding a common element of the fixed point set for two countable families of weak relatively nonexpansive mappings and the set of solutions of the system of generalized Ky Fan inequalities in a uniformly convex and uniformly smooth Banach space.

Motivated by the previously known results, we introduce a new hybrid iterative scheme of the generalized $f$-projection 
operator for finding a zero point of a maximal monotone operator in a Banach space. We prove that if $T^{-1} 0$ is nonempty, then our new iterative sequence converges strongly to an element of $T^{-1} 0$. As applications, we apply our results to obtain some new results for the convex minimization and the variational inequality problems in a Banach space. The results present in this paper are extension, improvement and generalization of the previously results.

\section{PRELIMINARIES}

Let $E$ be a real Banach space with dual $E^{*} E$ is said to be strictly convex if $\left\|\frac{x+y}{2}\right\|<1$ for all $x, y \in E$ with $\|x\|=\|y\|=1$ and $x \neq y$. The modulus of convexity of $E$ is the function $\delta:[0,2] \rightarrow[0,1]$ defined by

$\delta(\varepsilon)=\inf \left\{1-\left\|\frac{x+y}{2}\right\|: x, y \in E,\|x\|=\|y\|=1,\|x-y\| \geq \varepsilon\right\}$

A Banach space $E$ is said to be uniformly convex if and only if $\delta(\varepsilon)>0$ for all $\varepsilon \in(0,2]$.

Let $U=\{x \in E:\|x\|=1\}$ be the unit sphere of $E$. Then a Banach space $E$ is said to be smooth if the limit $\lim \frac{\|x+t y\|-\|x\|}{t}$ exists for each $x, y \in U . E$ is also said to be uniformly smooth if the limit exists uniformly in $x, y \in U$. Let $\langle\cdot, \cdot\rangle$ denote the duality pairing of $E^{*}$ and $E$, the normalized duality mapping $J: E \rightarrow 2^{E^{*}}$ is defined by

$$
J(x)=\left\{x^{*} \in E^{*}:\left\langle x, x^{*}\right\rangle=\|x\|^{2},\left\|x^{*}\right\|=\|x\|\right\} .
$$

If $E$ is a Hilbert space, then $J=I$, where $I$ is the identity mapping and $\langle\cdot, \cdot\rangle$ denotes an inner product on $E$. Consider the functional defined by

$$
\phi(x, y)=\|x\|^{2}-2\langle x, J y\rangle+\|y\|^{2}, \quad \text { for } x, y \in E,
$$

where $J: E \rightarrow 2^{E^{*}}$ is the normalized duality mapping.

As well know that if $C$ is a nonempty closed convex subset of a Hilbert space $H$ and $P_{C}: H \rightarrow C$ is the metric projection of $H$ onto $C$. This fact actually characterizes Hilbert spaces and consequently, it is not available in more general Banach spaces. In this connection, Alber [6], [7] recently introduced the generalized projection $\Pi_{C}: E \rightarrow C$ is a map that assigns to an arbitrary point $x \in E$ the minimum point of the functional $\phi(x, y)$, that is, $\Pi_{C} x=\bar{x}$, where $\bar{x}$ is the solution to the minimization problem

$$
\phi(\bar{x}, x)=\inf _{y \in C} \phi(y, x) .
$$

The existence and uniqueness of the operator $\Pi_{C}$ follows from the properties of the functional $\phi(y, x)$ and strict monotonicity of the mapping $J$ (see, for example, [6], [8], [17], [16]). It is obvious from the definition of function $\phi$ that

$$
(\|y\|-\|x\|)^{2} \leq \phi(y, x) \leq(\|y\|+\|x\|)^{2}, \quad \forall x, y \in E .
$$

We also known that

$$
\phi(x, y)=\phi(x, z)+\phi(z, y)+2\langle x-z, J z-J y\rangle .
$$

If $E$ is a Hilbert space, then $\phi(y, x)=\|y-x\|^{2}$ and $\Pi_{C}$ becomes the metric projection of $E$ onto $C$.

Remark 1: Let $E$ be a Banach space. Then we know that

1. if $E$ is an arbitrary Banach space, then $J$ is monotone and bounded;
2. if $E$ is a strictly convex, then $J$ is strictly monotone;

3. if $E$ is a smooth, then $J$ is single valued and semicontinuous;

4. if $E$ is uniformly smooth, then $J$ is uniformly normto-norm continuous on each bounded subset of $E$;

5. if $E$ is reflexive, smooth and strictly convex, then the normalized duality mapping $J$ is single valued, one-to-one and onto;

6. if $E$ is uniformly smooth, then $E$ is smooth and reflexive;

7. $E$ is uniformly smooth if and only if $E^{*}$ is uniformly convex;

see [8] for more details.

Remark 2: If $E$ is a reflexive, strictly convex and smooth Banach space, then for $x, y \in E, \phi(x, y)=0$ if and only if $x=y$. It is sufficient to show that if $\phi(x, y)=0$ then $x=y$. From 3, we have $\|x\|=\|y\|$. This implies that $\langle x, J y\rangle=$ $\|x\|^{2}=\|J y\|^{2}$. From the definition of $J$, one has $J x=J y$. Therefore, we have $x=y$; see [8], [16] for more details.

Lemma 1: (Kamimura and Takahashi [17]). Let E be a uniformly convex and smooth Banach space and let $\left\{x_{n}\right\}$ and $\left\{y_{n}\right\}$ be two sequences of $E$. If $\phi\left(x_{n}, y_{n}\right) \rightarrow 0$ and either $\left\{x_{n}\right\}$ or $\left\{y_{n}\right\}$ is bounded, then $x_{n}-y_{n} \rightarrow 0$.

Let $G: C \times E^{*} \longrightarrow \mathbf{R} \cup\{+\infty\}$ be a functional defined as follows:

$$
G(y, \varpi)=\|y\|^{2}-2\langle y, \varpi\rangle+\|\varpi\|^{2}+2 \rho f(y),
$$

where $y \in C, \varpi \in E^{*}, \rho$ is positive number and $f$ : $C \rightarrow \mathbf{R} \cup\{+\infty\}$ is proper, convex and lower semicontinuous. From definitions of $G$ and $f$, it is easy to see the following properties:

1) $G(y, \varpi)$ is convex and continuous with respect to $\varpi$ when $y$ is fixed;

2) $G(y, \varpi)$ is convex and lower semicontinuous with respect to $y$ when $\varpi$ is fixed.

Let $E$ be a real Banach space with its dual $E^{*}$. Let $C$ be a nonempty closed convex subset of $E$. We say that $\pi_{C}^{f}: E^{*} \rightarrow$ $2^{C}$ is generalized $f$-projection operator if

$$
\pi_{C}^{f} \varpi=\left\{u \in C: G(u, \varpi)=\inf _{y \in C} G(y, \varpi), \forall \varpi \in E^{*}\right\} .
$$

Lemma 2: (Wu and Hung [10]). Let $E$ be a reflexive Banach space with its dual $E^{*}$ and $C$ be a nonempty closed convex subset of $E$. The following statements hold:

1) $\pi_{C}^{f} \varpi$ is nonempty closed convex subset of $C$ for all $\varpi \in E^{*}$;

2) if $E$ is smooth, then for all $\varpi \in E^{*}, x \in \pi_{C}^{f} \varpi$ if and only if

$$
\langle x-y, \varpi-J x\rangle+\rho f(y)-\rho f(x) \geq 0, \forall y \in C ;
$$

$3)$ if $E$ is strictly convex and $f: C \rightarrow \mathbf{R} \cup\{+\infty\}$ is positive homogeneous (i.e., $f(t x)=t f(x)$ for all $t>0$ such that $t x \in C$ where $x \in C$ ), then $\pi_{C}^{f}$ is single valued mapping. 
Fan et al. [11] show that the condition $f$ is positive homogeneous which appeared in [11, Lemma 2.1 (iii)] can be removed.

Lemma 3: (Fan et al. [11]). Let $E$ be a reflexive Banach space with its dual $E^{*}$ and $C$ be a nonempty closed convex subset of $E$. If $E$ is strictly convex, then $\pi_{C}^{f} \varpi$ is single valued.

Recall that $J$ is single value mapping when $E$ is a smooth Banach space. There exists a unique element $\varpi \in E^{*}$ such that $\varpi=J x$ where $x \in E$. This substitution for (7) give

$$
G(y, J x)=\|y\|^{2}-2\langle y, J x\rangle+\|x\|^{2}+2 \rho f(y)
$$

It is obvious from the definition of $G$ that

$$
G(y, J x)=G(y, J z)+\phi(z, x)+2\langle y-z, J z-J x\rangle,
$$

for all $x, y, z \in E$.

Next, we consider the second generalized $f$-projection operator in Banach spaces.

Definition 1: (Li et al. [12]). Let $E$ be a real smooth Banach space and $C$ be a nonempty closed convex subset of $E$. We say that $\Pi_{C}^{f}: E \rightarrow 2^{C}$ is generalized $f$-projection operator if

$$
\Pi_{C}^{f} x=\left\{u \in C: G(u, J x)=\inf _{y \in C} G(y, J x)\right\}, \forall x \in E .
$$

Lemma 4: (Deimling [18]). Let $E$ be a Banach space and $f: E \rightarrow \mathbf{R} \cup\{+\infty\}$ be a lower semicontinuous convex functional. Then there exist $x^{*} \in E^{*}$ and $\alpha \in \mathbf{R}$ such that

$$
f(x) \geq\left\langle x, x^{*}\right\rangle+\alpha, \forall x \in E .
$$

Lemma 5: (Li et al. [12]). Let $E$ be a reflexive smooth Banach space and $C$ be a nonempty closed convex subset of $E$. The following statements hold:

1) $\Pi_{C}^{f} x$ is nonempty closed convex subset of $C$ for all $x \in E$;

2) for all $x \in E, \hat{x} \in \Pi_{C}^{f} x$ if and only if

$$
\langle\hat{x}-y, J x-J \hat{x}\rangle+\rho f(y)-\rho f(\hat{x}) \geq 0, \forall y \in C ;
$$

$3)$ if $E$ is strictly convex, then $\Pi_{C}^{f}$ is single valued mapping.

Lemma 6: (Li et al. [12]). Let $E$ be a reflexive smooth Banach space and $C$ be a nonempty closed convex subset of $E$ and let $x \in E, \hat{x} \in \Pi_{C}^{f} x$. Then

$$
\phi(y, \hat{x})+G(\hat{x}, J x) \leq G(y, J x), \forall y \in C .
$$

Remark 3: Let $E$ be a uniformly convex and uniformly smooth Banach space and $f(x)=0$ for all $x \in E$. Then Lemma 6 reduces to the property of the generalized projection operator considered by Alber [6].

Lemma 7: (Li et al. [12]). Let $E$ be a Banach space and $f$ : $E \rightarrow \mathbf{R} \cup\{+\infty\}$ be a proper, convex and lower semicontinuous mapping with convex domain $D(f)$. If $\left\{x_{n}\right\}$ is a sequence in $D(f)$ such that $x_{n} \rightarrow \hat{x} \in D(f)$ and $\lim _{n \rightarrow \infty} G\left(x_{n}, J y\right)=$ $G(\hat{x}, J y)$, then $\lim _{n \rightarrow \infty}\left\|x_{n}\right\|=\|\hat{x}\|$.

An operator $T$ is said to be closed if for any sequence $\left\{x_{n}\right\} \subset C$ with $x_{n} \rightarrow x$ and $T x_{n} \rightarrow y$, then $T x=y$.
An operator $T \subset E \times E^{*}$ is said to be monotone if $\langle x-$ $\left.y, x^{*}-y^{*}\right\rangle \geq 0$ whenever $\left(x, x^{*}\right),\left(y, y^{*}\right) \in T$. We denote the set $\{x \in E: 0 \in T x\}$ by $T^{-1} 0$. A monotone $T$ is said to be maximal if its graph $G(T)=\{(x, y): y \in T x\}$ is not properly contained in the graph of any other monotone operator. If $T$ is maximal monotone, then the solution set $T^{-1} 0$ is closed and convex. Let $E$ be a reflexive, strictly convex and smooth Banach space, it is knows that $T$ is a maximal monotone if and only if $R(J+r T)=E^{*}$ for all $r>0$. Define the resolvent of $T$ by $J_{r}=(J+r T)^{-1} J$ for all $r>0 . J_{r}$ is a single-valued mapping from $E$ to $D(T)$. Also, $T^{-1}(0)=F\left(J_{r}\right)$ for all $r>0$, where $F\left(J_{r}\right)$ is the set of all fixed points of $J_{r}$. Define, for $r>0$, the Yosida approximation of $T$ by $T_{r}=\left(J-J J_{r}\right) / r$. We know that $T_{r} x \in T\left(J_{r} x\right)$ for all $r>0$ and $x \in E$.

Lemma 8: (Kohsaka and Takahashi [13]). Let $E$ be a smooth, strictly convex and reflexive Banach space, let $C$ be a nonempty closed convex subset of $E$ and let $T \subset E \times E^{*}$ be a monotone operator satisfying $D(T) \subset C \subset J^{-1}\left(\cap_{r>0} R(J+\right.$ $r T)$ ). Let $r>0$, let $J_{r}$ and $T_{r}$ be the resolvent and the Yosida approximation of $T$, respectively. Then the following hold:

(i) $\phi\left(u, J_{r} x\right)+\phi\left(J_{r} x, x\right) \leq \phi(u, x), \forall x \in C, u \in T^{-1} 0$;

(ii) $\left(J_{r} x, T_{r} x\right) \in T, \forall x \in C$.

Lemma 9: Let $E$ be a smooth, strictly convex and reflexive Banach space, let $T \subset E \times E^{*}$ be a monotone operator with $T^{-1} 0 \neq \emptyset$, and let $J_{r}=(J+r T)^{-1} J$ for each $r>0$. Then

$$
G\left(p, J J_{r} x\right)+\phi\left(J_{r} x, x\right) \leq G(p, J x), \forall x \in E, p \in T^{-1} 0 .
$$

Proof. Let $r>0, p \in T^{-1} 0$, and $x \in E$. By the monotonicity of $T$ and (9), we have

$$
\begin{aligned}
G(p, J x) & =G\left(p, J J_{r} x\right)+\phi\left(J_{r} x, x\right)+2\left\langle p-J_{r} x, J J_{r} x-J x\right\rangle \\
& =G\left(p, J J_{r} x\right)+\phi\left(J_{r} x, x\right)+2 r\left\langle p-J_{r} x,-T_{r} x\right\rangle \\
& \geq G\left(p, J J_{r} x\right)+\phi\left(J_{r} x, x\right) .
\end{aligned}
$$

The proof is complete.

\section{MAIN RESULTS}

Theorem 1: Let $C$ be a nonempty closed convex subset of a uniformly convex and uniformly smooth Banach space $E$. Let $T \subset E \times E^{*}$ be a maximal monotone operator satisfying $D(T) \subset C$ and let $J_{r_{n}}=\left(J+r_{n} T\right)^{-1} J$ for all $r_{n}>0$. Let $f: E \rightarrow \mathbf{R}$ be a convex and lower semicontinuous function with $C \subset \operatorname{int}(D(f))$. Assume that $T^{-1} 0 \neq \emptyset$, for arbitrary point $x_{1} \in C_{1}$ with $C_{1}=C$, generate a sequences $\left\{x_{n}\right\}$ by

$$
\left\{\begin{array}{l}
y_{n}=J^{-1}\left(\alpha_{n} J x_{n}+\left(1-\alpha_{n}\right) J J_{r_{n}} x_{n}\right), \\
C_{n+1}=\left\{z \in C_{n}: G\left(z, J y_{n}\right) \leq G\left(z, J x_{n}\right)\right\} \\
x_{n+1}=\Pi_{C_{n+1}}^{f} x_{1}
\end{array}\right.
$$

where $\left\{\alpha_{n}\right\}$ is a sequence in $[0,1]$ and $\left\{r_{n}\right\}$ is a sequence in $(0, \infty)$. If $\liminf _{n \rightarrow \infty}\left(1-\alpha_{n}\right)>0$ and $\lim _{n \rightarrow \infty} r_{n}=\infty$, then $\left\{x_{n}\right\}$ converges strongly to $\Pi_{T^{-1} 0}^{f} x_{1}$.

Proof. We first show that $C_{n+1}$ is closed and convex for each $n \geq 1$. Clearly $C_{1}=C$ is closed and convex. Suppose that $C_{n}$ is closed and convex for each $n \in \mathbf{N}$. Since for any $z \in C_{n}$, we know that $G\left(z, J y_{n}\right) \leq G\left(z, J x_{n}\right)$ is equivalent to

$$
2\left\langle z, J x_{n}-J y_{n}\right\rangle \leq\left\|x_{n}\right\|^{2}-\left\|y_{n}\right\|^{2} .
$$


Therefore, $C_{n+1}$ is closed and convex. This implies that $\Pi_{C_{n+1}}^{f} x_{1}$ is well defined.

Next, we will show by induction that $T^{-1} 0 \subset C_{n}$ for all $n \in \mathbf{N}$. It is obvious that $T^{-1} 0 \subset C_{1}=C$. Suppose that $T^{-1} 0 \subset C_{n}$ for some $n \in \mathbf{N}$. Let $q \in T^{-1} 0$, put $v_{n}=J_{r_{n}} x_{n}$ for all $n \geq 1$ and by lemma 9 , we have

$$
\begin{aligned}
G\left(q, J y_{n}\right)= & G\left(q, \alpha_{n} J x_{n}+\left(1-\alpha_{n}\right) J v_{n}\right) \\
= & \|q\|^{2}-2\left\langle q, \alpha_{n} J x_{n}+\left(1-\alpha_{n}\right) J v_{n}\right\rangle \\
& +\left\|\alpha_{n} J x_{n}+\left(1-\alpha_{n}\right) J v_{n}\right\|^{2}+2 \rho f(q) \\
\leq & \|q\|^{2}-2 \alpha_{n}\left\langle q, J x_{n}\right\rangle-2\left(1-\alpha_{n}\right)\left\langle q, J v_{n}\right\rangle \\
& +\alpha_{n}\left\|J x_{n}\right\|^{2}+\left(1-\alpha_{n}\right)\left\|J v_{n}\right\|^{2}+2 \rho f(q) \\
= & \alpha_{n} G\left(q, J x_{n}\right)+\left(1-\alpha_{n}\right) G\left(q, J v_{n}\right) \\
= & \alpha_{n} G\left(q, J x_{n}\right)+\left(1-\alpha_{n}\right) G\left(q, J J_{r_{n}} x_{n}\right) \\
\leq & \alpha_{n} G\left(q, J x_{n}\right)+\left(1-\alpha_{n}\right) G\left(q, J x_{n}\right) \\
= & G\left(q, J x_{n}\right) .
\end{aligned}
$$

So, $p \in C_{n+1}$. That is $T^{-1} 0 \in C_{n+1}$. Consequently, $T^{-1} 0 \subset C_{n}$, for all $n \in \mathbf{N}$. This implies that $\left\{x_{n}\right\}$ is well defined. Since $f: E \rightarrow \mathbf{R}$ is convex and lower semicontinuous mapping, from Lemma 4, we known that there exist $x^{*} \in E^{*}$ and $\alpha \in \mathbf{R}$ such that

$$
f(x) \geq\left\langle x, x^{*}\right\rangle+\alpha, \forall x \in E .
$$

For $x_{n} \in E$, it follows that

$$
\begin{aligned}
G\left(x_{n}, J x_{1}\right)= & \left\|x_{n}\right\|^{2}-2\left\langle x_{n}, J x_{1}\right\rangle+\left\|x_{1}\right\|^{2}+2 \rho f\left(x_{n}\right) \\
\geq & \left\|x_{n}\right\|^{2}-2\left\langle x_{n}, J x_{1}\right\rangle+\left\|x_{1}\right\|^{2}+2 \rho\left\langle x_{n}, x^{*}\right\rangle \\
& +2 \rho \alpha \\
= & \left\|x_{n}\right\|^{2}-2\left\langle x_{n}, J x_{1}-\rho x^{*}\right\rangle+\left\|x_{1}\right\|^{2} \\
& +2 \rho \alpha \\
\geq & \left\|x_{n}\right\|^{2}-2\left\|x_{n}\right\|\left\|J x_{1}-\rho x^{*}\right\|+\left\|x_{1}\right\|^{2} \\
& +2 \rho \alpha \\
= & \left(\left\|x_{n}\right\|-\left\|J x_{1}-\rho x^{*}\right\|\right)^{2}+\left\|x_{1}\right\|^{2} \\
& -\left\|J x_{1}-\rho x^{*}\right\|^{2}+2 \rho \alpha .
\end{aligned}
$$

For each $q \in T^{-1} 0 \subset C_{n}$ and $x_{n}=\Pi_{C_{n}}^{f} x_{1}$, by the definition of $C_{n}$ it follows from (13) that

$$
\begin{aligned}
G\left(q, J x_{1}\right) \geq & \\
\geq & G\left(x_{n}, J x_{1}\right) \\
\geq & \left(\left\|x_{n}\right\|-\left\|J x_{1}-\rho x^{*}\right\|\right)^{2}+\left\|x_{1}\right\|^{2} \\
& -\left\|J x_{1}-\rho x^{*}\right\|^{2}+2 \rho \alpha .
\end{aligned}
$$

This implies that $\left\{x_{n}\right\}$ is bounded and so are $\left\{y_{n}\right\}$ and $\left\{G\left(x_{n}, J x_{1}\right)\right\}$.

By the fact that $x_{n+1}=\Pi_{C_{n+1}}^{f} x_{1} \in C_{n+1} \subset C_{n}$ and $x_{n}=\Pi_{C_{n}}^{f} x_{1}$, it follows by Lemma 6 , we get

$$
\begin{aligned}
0 & \leq\left(\left\|x_{n+1}-\right\| x_{n} \|\right)^{2} \\
& \leq \phi\left(x_{n+1}, x_{n}\right) \\
& \leq G\left(x_{n+1}, J x_{1}\right)-G\left(x_{n}, J x_{1}\right)
\end{aligned}
$$

This implies that $\left\{G\left(x_{n}, J x_{1}\right)\right\}$ is nondecreasing. So, $\lim _{n \rightarrow \infty} G\left(x_{n}, J x_{1}\right)$ exist.

For any $m>n, x_{n}=\Pi_{C_{n}}^{f} x_{1}, x_{m}=\Pi_{C_{m}}^{f} x_{1} \in C_{m} \subset C_{n}$ and from (14), we have

$$
\phi\left(x_{m}, x_{n}\right) \leq G\left(x_{m}, J x_{1}\right)-G\left(x_{n}, J x_{1}\right) .
$$

Taking $m, n \rightarrow \infty$, we have $\phi\left(x_{m}, x_{n}\right) \rightarrow 0$. From Lemma 1 we get that $\left\|x_{n}-x_{m}\right\| \rightarrow 0$. Hence, $\left\{x_{n}\right\}$ is a Cauchy sequence. Since $E$ is a Banach space and $C_{n}$ is closed and convex, we can assume that there exists $p \in C$ such that $x_{n} \rightarrow$ $p \in C$ as $x_{n} \rightarrow \infty$. In particular, since $\lim _{n \rightarrow \infty} G\left(x_{n}, J x_{1}\right)$ exist from (14), we also have

$$
\lim _{n \rightarrow \infty} \phi\left(x_{n+1}, x_{n}\right)=0 .
$$

It follow from Lemma 1 that

$$
\lim _{n \rightarrow \infty}\left\|x_{n+1}-x_{n}\right\|=0 .
$$

Since $J$ is uniformly norm-to-norm continuous on bounded subsets of $E$, we obtain that

$$
\lim _{n \rightarrow \infty}\left\|J x_{n+1}-J x_{n}\right\|=0 .
$$

From definition of $C_{n+1}$ and $x_{n+1}=\Pi_{C_{n+1}}^{f} x_{1}$, we have

$$
G\left(x_{n+1}, J y_{n}\right) \leq G\left(x_{n+1}, J x_{n}\right) .
$$

Therefore, we obtain that

$$
\begin{array}{r}
\left\|x_{n+1}\right\|^{2}-2\left\langle x_{n+1}, J y_{n}\right\rangle+\left\|y_{n}\right\|^{2}+2 \rho f\left(x_{n+1}\right) \\
\leq\left\|x_{n+1}\right\|^{2}-2\left\langle x_{n+1}, J x_{n}\right\rangle+\left\|x_{n}\right\|^{2}+2 \rho f\left(x_{n+1}\right)
\end{array}
$$

then

$$
\begin{array}{r}
\left\|x_{n+1}\right\|^{2}-2\left\langle x_{n+1}, J y_{n}\right\rangle+\left\|y_{n}\right\|^{2} \\
\leq\left\|x_{n+1}\right\|^{2}-2\left\langle x_{n+1}, J x_{n}\right\rangle+\left\|x_{n}\right\|^{2}
\end{array}
$$

so,

$$
\phi\left(x_{n+1}, y_{n}\right) \leq \phi\left(x_{n+1}, x_{n}\right) .
$$

From Lemma 1 and (15), it follows that

$$
\lim _{n \rightarrow \infty}\left\|x_{n+1}-y_{n}\right\|=0
$$

and

$$
\lim _{n \rightarrow \infty}\left\|J x_{n+1}-J y_{n}\right\|=0
$$

From (12), we have

$$
G\left(q, J v_{n}\right) \geq \frac{1}{1-\alpha_{n}}\left(G\left(q, J y_{n}\right)-\alpha_{n} G\left(q, J x_{n}\right)\right)
$$

and from Lemma 9, we observe that

$$
\begin{aligned}
\phi\left(v_{n}, x_{n}\right) \leq & \phi\left(J_{r_{n}} x_{n}, x_{n}\right) \\
\leq & G\left(q, J x_{n}\right)-G\left(q, J J_{r_{n}} x_{n}\right) \\
= & G\left(q, J x_{n}\right)-G\left(q, J v_{n}\right) \\
\leq & G\left(q, J x_{n}\right)-\frac{1}{1-\alpha_{n}}\left(G\left(q, J y_{n}\right)-\alpha_{n} G\left(q, J x_{n}\right)\right. \\
= & \frac{1}{1-\alpha_{n}}\left(G\left(q, J x_{n}\right)-G\left(q, J y_{n}\right)\right) \\
= & \frac{1}{1-\alpha_{n}}\left(\left\|x_{n}\right\|^{2}-\left\|y_{n}\right\|^{2}-2\left\langle q, J x_{n}-J y_{n}\right\rangle\right) \\
\leq & \frac{1}{1-\alpha_{n}}\left(\left(\left\|x_{n}\right\|^{2}-\left\|y_{n}\right\|^{2}\right)+2 \mid\left\langle q, J x_{n}-J y_{n}\right\rangle\right) \\
\leq & \frac{1}{1-\alpha_{n}}\left(\left(\left\|x_{n}\right\|-\left\|y_{n}\right\|\right)\left(\left\|x_{n}\right\|+\left\|y_{n}\right\|\right)\right. \\
& \left.+2\|q\|\left\|J x_{n}-J y_{n}\right\|\right) \\
\leq & \frac{1}{1-\alpha_{n}}\left(\left(\left\|x_{n}\right\|-\left\|y_{n}\right\|\right)\left(\left\|x_{n}\right\|+\left\|y_{n}\right\|\right)\right. \\
& \left.+2\|q\|\left\|J x_{n}-J y_{n}\right\|\right) .
\end{aligned}
$$

Applied from (18), (19) and $\liminf _{n \rightarrow \infty}\left(1-\alpha_{n}\right)>0$, we get

$$
\lim _{n \rightarrow \infty} \phi\left(v_{n}, x_{n}\right)=0 .
$$


From Lemma 1, we get

$$
\lim _{n \rightarrow \infty}\left\|v_{n}-x_{n}\right\|=0 .
$$

Since $J$ is uniformly norm-to-norm continuous, we have

$$
\lim _{n \rightarrow \infty}\left\|J x_{n}-J v_{n}\right\|=0 .
$$

Noticing the condition $r_{n}>0$, it follows that

$$
\lim _{n \rightarrow \infty} \frac{1}{r_{n}}\left\|J x_{n}-J v_{n}\right\|=0 .
$$

Therefore,

$$
\begin{aligned}
& \lim _{n \rightarrow \infty}\left\|T_{r_{n}} x_{n}\right\|=\lim _{n \rightarrow \infty} \frac{1}{r_{n}}\left\|J x_{n}-J J_{r_{n}} x_{n}\right\| \\
& =\lim _{n \rightarrow \infty} \frac{1}{r_{n}}\left\|J x_{n}-J v_{n}\right\|=0 .
\end{aligned}
$$

For $\left(w, w^{*}\right) \in T$, from the monotonicity of $T$, we have $\langle w-$ $\left.v_{n}, w^{*}-T_{r_{n}} x_{n}\right\rangle \geq 0$ for all $n \geq 0$. Letting $n \rightarrow \infty$, we get $\left\langle w-p, w^{*}\right\rangle \geq 0$. From the maximality of $T$, we have $p \in$ $T^{-1} 0$. Finally, we show that $p=\Pi_{T^{-1} 0}^{f} x_{1}$. Since $F$ is closed and convex set from Lemma 5 , we have $\Pi_{T^{-1} 0}^{f} x_{1}$ is single value, denote by $v$. From $x_{n}=\Pi_{C_{n}}^{f} x_{1}$ and $v \in F \subset C_{n}$, we also have

$$
G\left(x_{n}, J x_{1}\right) \leq G\left(v, J x_{1}\right), \forall n \geq 1 .
$$

By definition of $G$ and $f$, we know that, for each given $x$, $G(y, J x)$ is convex and lower semicontinuous with respect to $y$. So

$$
\begin{aligned}
G\left(p, J x_{1}\right) & \\
& \leq \liminf _{n \rightarrow \infty} G\left(x_{n}, J x_{1}\right) \\
& \leq \limsup _{n \rightarrow \infty} G\left(x_{n}, J x_{1}\right) \\
& \leq G\left(v, J x_{1}\right) .
\end{aligned}
$$

From definition of $\Pi_{T^{-1} 0}^{f} x_{1}$ and $p \in T^{-1} 0$, we can conclude that $v=p=\Pi_{T^{-1} 0}^{f} x_{1}$ and $x_{n} \rightarrow p$ as $n \rightarrow \infty$. This completes the proof.

Taking $f(y)=0$ for all $y \in E$, we have $G(y, J x)=$ $\phi(y, x)$ and $\Pi_{C}^{f} x=\Pi_{C} x$. From Theorem 1 we obtain the following corollary.

Corollary 1: Let $C$ be a nonempty closed convex subset of a uniformly convex and uniformly smooth Banach space $E$. Let $T \subset E \times E^{*}$ be a maximal monotone operator satisfying $D(T) \subset C$ and let $J_{r_{n}}=\left(J+r_{n} T\right)^{-1} J$ for all $r_{n}>0$. Assume that $T^{-1} 0 \neq \emptyset$. For arbitrary point $x_{1} \in C_{1}$ with $C_{1}=C$, generate a sequences $\left\{x_{n}\right\}$ by

$$
\left\{\begin{array}{l}
y_{n}=J^{-1}\left(\alpha_{n} J x_{n}+\left(1-\alpha_{n}\right) J J_{r_{n}} x_{n}\right) \\
C_{n+1}=\left\{z \in C_{n}: \phi\left(z, J y_{n}\right) \leq \phi\left(z, J x_{n}\right)\right\} \\
x_{n+1}=\prod_{C_{n+1}} x_{1}
\end{array}\right.
$$

where $\left\{\alpha_{n}\right\}$ is a sequence in $[0,1]$ and $\left\{r_{n}\right\}$ is a sequence in $(0, \infty)$. If $\liminf _{n \rightarrow \infty}\left(1-\alpha_{n}\right)>0$ and $\lim _{n \rightarrow \infty} r_{n}=\infty$, then $\left\{x_{n}\right\}$ converges strongly to $\Pi_{T^{-1} 0} x_{1}$.

\section{APPLICATIONS}

\section{A. Convex minimization problem}

In this section, we study the problem for finding a minimizer of a proper lower semicontinuous convex function in Banach spaces.

if

A proper function $F: E \rightarrow(-\infty, \infty]$ is said to be convex

$$
F(\alpha x+(1-\alpha) y) \leq \alpha F(x)+(1-\alpha) F(y),
$$

for all $x, y \in E$ and $\alpha \in(0,1)$.

The function $F$ is said to be lower semicontinuous if the set $\{x \in E: F(x) \leq r\}$ is closed in $E$ for all $r \in \mathbf{R}$. For a proper lower semicontinuous convex function $F: E \rightarrow(-\infty, \infty]$, the subdifferential $\partial F$ of $F$ is defined by

$\partial F(x)=\left\{x^{*} \in E^{*}: F(x)+\left\langle y-x, x^{*}\right\rangle \leq F(y), \forall y \in E\right\}$,

for all $x \in E$. It is easy to see that $0 \in \partial F(u)$ if and only if $F(u)=\min _{x \in E} F(x)$. Rockafellar [20] proved that the subdifferential mapping $\partial F \subset E \times E^{*}$ of $F$ is a maximal monotone operator.

Lemma 10: ( Takahashi [21]) Let $E$ be a Banach spaces, let $F \rightarrow(-\infty, \infty]$ be a proper lower semicontinuous convex function and let $g: E \rightarrow \mathbf{R}$ be a continuous convex function.Then

$$
\partial(F+g)(x)=\partial F(x)+\partial g(x),
$$

for all $x \in E$.

Theorem 2: Let $C$ be a nonempty closed convex subset of a uniformly convex and uniformly smooth Banach space $E$. Let $F \rightarrow(-\infty, \infty]$ be a proper lower semicontinuous convex function and let $f: E \rightarrow \mathbf{R}$ be a convex and lower semicontinuous function with $C \subset \operatorname{int}(D(f))$. Assume that $(\partial F)^{-1} 0 \neq \emptyset$, for arbitrary point $x_{1} \in C_{1}$ with $C_{1}=C$, generate a sequences $\left\{x_{n}\right\}$ by

$$
\left\{\begin{array}{l}
z_{n}=\operatorname{argmin}_{u \in E} F(u)+\frac{1}{2 r_{n}}\|u\|^{2}-\frac{1}{r_{n}}\left\langle u, J x_{n}\right\rangle \\
y_{n}=J^{-1}\left(\alpha_{n} J x_{n}+\left(1-\alpha_{n}\right) J z_{n}\right), \\
C_{n+1}=\left\{z \in C_{n}: G\left(z, J y_{n}\right) \leq G\left(z, J x_{n}\right)\right\}, \\
x_{n+1}=\prod_{C_{n+1}}^{f} x_{1}
\end{array}\right.
$$

where $\left\{\alpha_{n}\right\}$ is a sequence in $[0,1]$ and $\left\{r_{n}\right\} \subset(0, \infty)$. If $\liminf _{n \rightarrow \infty}\left(1-\alpha_{n}\right)>0$ and $\lim _{n \rightarrow \infty} r_{n}=\infty$, then $\left\{x_{n}\right\}$ converges strongly to $\Pi_{(\partial F)^{-1} 0}^{f} x_{1}$.

Proof. We known that $(\partial F)^{-1} 0$ is a maximal monotone operator. For $w \in E$ and $r>0$, let $J_{r}$ be the resolvent of $(\partial F)$, we have

$$
J w \in J\left(J_{r} w\right)+r(\partial F)\left(J_{r} w\right) .
$$

Hence

$0 \in(\partial F)\left(J_{r} w\right)+\frac{1}{r} J\left(J_{r} w\right)-\frac{1}{r} J w=\partial\left(F+\frac{1}{2 r}\|\cdot\|^{2}-\frac{1}{r} J w\right)\left(J_{r} w\right)$

that is

$$
J_{z} w=\operatorname{argmin}_{u \in E}\left\{F(u)+\frac{1}{2 r}\|u\|^{2}-\frac{1}{r}\langle u, J w\rangle\right\} .
$$

Since $z_{n}=J_{r_{n}} x_{n}$ for all $n=1,2,3, \ldots$. By Theorem $1,\left\{x_{n}\right\}$ converges strongly to $\Pi_{(\partial F)^{-1} 0}^{f} x_{1}$. 


\section{B. Variational inequality problem}

Let $E$ be a real Banach space and let $C$ be a nonempty closed and convex subset of $E$ and $A: C \rightarrow E^{*}$ be an operator. The variational inequality problem for an operator $\mathrm{A}$ is to find $x \in C$ such that

$$
\langle y-x, A x,\rangle \geq 0, \quad \forall y \in C .
$$

The set of solution of 31 is denote by $V I(A, C)$.

Let $A$ be a monotone mapping of $C$ into $E^{*}$ which is said to be hemicontinuous if for all $x, y \in C$, the mapping $h$ of $[0,1]$ into $E^{*}$, defined by $h(t)=A(t x+(1-t) y)$, is continuous with respect to the weak* topology of $E^{*}$. We define by $N_{C}(v)$ the normal cone for $C$ at a point $v \in C$, that is,

$$
N_{C}(v)=\left\{x^{*} \in E^{*}:\left\langle y-v, x^{*}\right\rangle \leq 0, \quad \forall y \in C\right\} .
$$

Lemma 11: (Rockafellar [19]). Let $C$ be a nonempty, closed convex subset of a Banach space $E$ and $A$ a monotone, hemicontinuous operator of $C$ into $E^{*}$. Let $T \subset E \times E^{*}$ be an operator defined as follows:

$$
T v=\left\{\begin{array}{l}
A v+N_{C}(v), \quad v \in C \\
\emptyset, \text { otherwise. }
\end{array}\right.
$$

Then $T$ is maximal monotone and $T^{-1} 0=V I(A, C)$.

Theorem 3: Let $C$ be a nonempty closed and convex subset of a uniformly convex and uniformly smooth Banach space $E$. Let $A: C \rightarrow E^{*}$ be a single-value, monotone and hemicontinuous operator and let $f: E \rightarrow \mathbf{R}$ be a convex and lower semicontinuous function with $C \subset \operatorname{int}(D(f))$. Assume that $V I(A, C) \neq \emptyset$. For arbitrary point $x_{1} \in C_{1}$ with $C_{1}=C$, generate a sequences $\left\{x_{n}\right\}$ by

$$
\left\{\begin{array}{l}
z_{n}=V I\left(A+\frac{1}{r_{n}}\left(J-J x_{n}\right), C\right), \\
y_{n}=J^{-1}\left(\alpha_{n} J x_{n}+\left(1-\alpha_{n}\right) J z_{n}\right), \\
C_{n+1}=\left\{z \in C_{n}: G\left(z, J y_{n}\right) \leq G\left(z, J x_{n}\right)\right\}, \\
x_{n+1}=\Pi_{C_{n+1}}^{f} x_{1}, n=1,2,3, \ldots,
\end{array}\right.
$$

where $\left\{\alpha_{n}\right\}$ is a sequence in $[0,1]$ and $\left\{r_{n}\right\} \subset(0, \infty)$. If $\liminf _{n \rightarrow \infty}\left(1-\alpha_{n}\right)>0$ and $\lim _{n \rightarrow \infty} r_{n}=\infty$, then $\left\{x_{n}\right\}$ converges strongly to $\Pi_{V I(A, C)}^{f} x_{1}$.

Proof. For $w \in E$ and $r>0$, by Theorem 11, we have

$$
J w \in J\left(J_{r} w\right)+r T\left(J_{r} w\right) .
$$

Hence

$$
-A\left(J_{r} w\right)+\frac{1}{r}\left(J w-J\left(J_{r} w\right)\right) \in N_{C}\left(J_{r} w\right) .
$$

It follows that $\left\langle y-J_{r} w, A\left(J_{r} w\right)+\frac{1}{r}\left(J\left(J_{r} w\right)-J w\right)\right\rangle \geq 0$, for all $y \in C$. That is $J_{r} w=V I\left(A+\frac{1}{r}(J-J w), C\right)$. Since $z_{n}=$ $J_{r_{n}} x_{n}$ for all $n=1,2,3, \ldots$, by Theorem $1,\left\{x_{n}\right\}$ converges strongly to $\Pi_{V I(A, C)}^{f} x_{1}$.

\section{CONCLUSION}

In this paper, we extend and improve the iterative scheme for resolvents of maximal monotone operators from using the generalized projection to using the generalized $f$-projection. We apply this result to the convex minimization and variational inequality problems in Banach spaces.

\section{ACKNOWLEDGMENT} Fund.

This work was supported by Thaksin University Research

\section{REFERENCES}

[1] B. Martinet, Régularisation d inéquations variationelles par approximations succesives. Rev. Fr. Inf. Rech. Opér. 2, 154-159 (1970).

[2] R.T. Rockafellar, Monotone operators and the proximal point algorithm, SIAM J. Control Optim. 14 (1976) 877-898.

[3] O. G Güler, On the convergence of the proximal point algorithm for convex minimization, SIAMJ. Control Optim. 29 (1991), no. 2, 403419.

[4] M. V. Solodov and B. F. Svaiter, Forcing strong convergence of proximal point iterations in a Hilbert space, Math.Program.87 (2000), 189-202.

[5] S. Kamimura and W. Takahashi, Approximating solutions of maximal monotone operators in Hilbert spaces, J.Approx.Theory106 (2000), no. 2, 226-240.

[6] Ya. Alber, Metric and generalized projection operators in Banach spaces: Properties and applications, in: A. Kartsatos (Ed.), Theory and Applications of Nonlinear Operators of Accretive and Monotone Type, Dekker, New York, 1996, pp. 15-50.

[7] Ya. Alber, Generalized projection operators in Banach spaces: Properties and applications, in: Proceedings of the Israel Seminar, Ariel, Israel, Funct. Differential Equation 1 (1994) 1-21.

[8] I. Cioranescu, Geometry of Banach spaces, Duality Mappings and Nonlinear Problems, Kluwer, Dordrecht, 1990.

[9] J. L. Li, The generalized projection operator on reflexive Banach spaces and its applications, J. Math. Anal. Appl. 306 (2005) 55-71.

[10] K. Q. Wu, N. J. Huang, The generalised $f$-projection operator with an application, Bull. Aust. Math. Soc. 73(2006) 307-317.

[11] J.H. Fan, X. Liu, J.L. Li, Iterative schemes for approximating solutions of generalized variational inequalities in Banach spaces, Nonlinear Anal. TMA 70(2009) 3997-4007.

[12] X. Li, N. Huang, D. O'Regan, Strong convergence theorems for relatively nonexpansive mappings in Banach spaces with applications, Comp. Math. Appl. 60(2010), 1322-1331.

[13] F. Kohsaka and W. Takahashi, Strong convergence of an iterative sequence for maximal monotone operators in a Banach space, Abstract and Applied Analysis, vol. 2004, no. 3,239-249.

[14] S. Matsushita, W. Takahashi, A strong convergence theorem for relatively nonexpansive mappings in a Banach space, J. Approx. Theory 134 (2005) 257-266.

[15] S. Saewan and P. Kumam, A generalized f-projection method for countable families of weak relatively nonexpansive mappings and the system of generalized Ky Fan inequalities, J Glob Optim, DOI 10.1007/s10898-012-9922-3.

[16] W. Takahashi, Nonlinear Functional Analysis, Yokohama-Publishers, 2000 .

[17] S. Kamimura, W. Takahashi, Strong convergence of a proximal-type algorithm in a Banach space, SIAM J. Optim. 13 (2002) 938-945.

[18] K. Deimling, Nonlinear Functional Analysis, Springer-Verlag, Berlin, Heidelberg, New York, Tokyo, 1985.

[19] R. T. Rockafellar, On the maximality of sums of nonlinear monotone operators, Trans. Amer. Math. Soc 149(1970), 75-88.

[20] R. T. Rockafellar, Characterization of the subdifferentials of convex functions, Pacific J. Math. 17 (1966), 497-510.

[21] W. Takahashi, Totsu Kaiseki to Fudōten Kinji [Convex Analysis and Approximation of Fixed Points, Sūrikaiseki Shiriizu, vol. 2, Yokohama Publishers, Yokohama, 2000.

\section{Creative Commons Attribution License 4.0 (Attribution 4.0 International, CC BY 4.0)}

This article is published under the terms of the Creative Commons Attribution License 4.0 https://creativecommons.org/licenses/by/4.0/deed.en US 